Climate Change Economics, Vol. 8, No. 2 (2017) 1750009 (28 pages)

(C) The Author(s)

DOI: $10.1142 / \mathrm{S} 2010007817500099$

\title{
UNDERSTANDING THE SOCIAL COST OF CARBON: A MODEL DIAGNOSTIC AND INTER-COMPARISON STUDY*
}

\author{
STEVEN K. ROSE ${ }^{\dagger}$, DELAVANE B. DIAZ and GEOFFREY J. BLANFORD \\ Energy and Environmental Analysis Research Group \\ Electric Power Research Institute (EPRI) \\ Palo Alto, CA 94304, USA \\ †rose@epri.com
}

Accepted 12 May 2017

Published 9 June 2017

\begin{abstract}
The social cost of carbon (SCC) is a monetary estimate of global climate change damages to society from an additional unit of carbon dioxide $\left(\mathrm{CO}_{2}\right)$ emissions. SCCs are used to estimate the benefits of $\mathrm{CO}_{2}$ reductions from policies. However, little is known about the modeling underlying the values or the implied societal risks, making SCC estimates difficult to interpret and assess. This study performs the first in-depth examination of SCC modeling using controlled diagnostic experiments that yield detailed intermediate results, allow for direct comparison of individual components of the models, and facilitate evaluation of the individual model SCCs. Specifically, we analyze DICE, FUND, and PAGE and the multimodel approach used by the US Government. Through our component assessments, we trace SCC differences back to intermediate variables and specific features. We find significant variation in component-level behavior between models driven by model-specific structural and implementation elements, some resulting in artificial differences in results. These elements combine to produce model-specific tendencies in climate and damage responses that contribute to differences observed in SCC outcomes - producing PAGE SCC distributions with longer and fatter right tails and higher averages, followed by DICE with more compact distributions and lower averages, and FUND with distributions that include net benefits and the lowest averages. Overall, our analyses reveal fundamental model behavior relevant to many disciplines of climate research, and identify issues with the models, as well as the overall multimodel approach, that need further consideration. With the growing prominence of SCCs in decision-making, ranging from the local-level to international, improved transparency and technical understanding is essential for informed decisions.
\end{abstract}

Keywords: Social cost of carbon; social cost of greenhouse gases; climate change; carbon cycle; impacts; damages.

\footnotetext{
*This article contains supplementary material (SM) available on the journal website. The supplementary information includes a modeling overview for the US Government approach, structural details for individual models, standardized inputs for our diagnostic experiments, additional results from our analyses, and a summary of the literature underlying damages specifications.

This is an Open Access article published by World Scientific Publishing Company. It is distributed under the terms of the Creative Commons Attribution 4.0 (CC-BY) License. Further distribution of this work is permitted, provided the original work is properly cited.
} 


\section{Introduction}

The social cost of carbon (SCC) is defined as the incremental monetized global damages arising from an additional unit of $\mathrm{CO}_{2}$ emitted to the atmosphere. It is also referred to as the social cost of $\mathrm{CO}_{2}$. It is socially important as an estimate of the global damages of $\mathrm{CO}_{2}$ emissions, and practically important for policy-making. For instance, United States Government (USG) agencies are now legally required to value $\mathrm{CO}_{2}$ emissions in rulemakings to assess the potential benefits of $\mathrm{CO}_{2}$ reductions from regulations, including rules affecting appliances, vehicles, and industry. ${ }^{1}$ Furthermore, SCC estimates, frequently the USG estimates, are increasingly being applied or considered at state (e.g., Colorado, Maine, Minnesota, New York) and local levels, as well as by other countries (e.g., Canada). The USG developed its own SCC estimates for use in rulemakings, first in 2010 and then revised in 2013 and 2015 with significantly higher estimates (USG Interagency Working Group on Social Cost of Carbon, 2010, 2013, 2015, 2016). However, little is known or available about the modeling underlying current estimates, making them difficult to interpret or assess.

The SCC is typically estimated using an integrated assessment model (IAM) to simulate a "causal chain," starting with projected socioeconomic futures, and their greenhouse gas emissions, followed by projected resulting climate change and climate damages for both a reference and a $\mathrm{CO}_{2}$ pulsed emissions trajectory (Fig. S1). The SCC associated with a $\mathrm{CO}_{2}$ pulse in a particular year is the discounted value of the future annual incremental damages off of the reference trajectory. To date, only a few IAMs, those with more aggregate economic structures and damage components, have generated SCC estimates.

The USG estimates were derived from a complex approach using three IAMs well known in the SCC literature: DICE (Nordhaus, 2010), FUND (Anthoff and Tol, 2013), and PAGE (Hope, 2011). While each of these models has been applied individually to estimate the SCC - indeed, most published estimates are from versions of these models - the USG approach was novel in its experimental design, running multiple models tens of thousands of times each with standardized and model-specific uncertainties, standardized discounting, and a procedure for aggregating the results and selecting values. Through this approach, the USG produced SCC estimates to apply to the estimated emission changes in current and future years from 2010-2050. Underlying each official USG SCC is a wide range of estimates, with a frequency distribution of 150,000 estimates (50,000 from each model). For $\mathrm{CO}_{2}$ emitted in 2020, for instance, the current USG estimates have a central value of $\$ 42 / \mathrm{tCO}_{2}$ (\$2007), with alternate estimates of $\$ 12$ to $\$ 123 / \mathrm{tCO}_{2}$ corresponding to different discount rates and likelihoods, and behind each are 150,000 estimates (USG Interagency Working Group on Social Cost of Carbon, 2015, 2016). For an overview of the USG experimental design, see Table S1 in the Supplementary Material (SM).

\footnotetext{
${ }^{1}$ Center for Biological Diversity versus National Highway Traffic Safety Administration, United States Court of Appeals for the Ninth Circuit, No. 06-71891, November 15, 2007.
} 


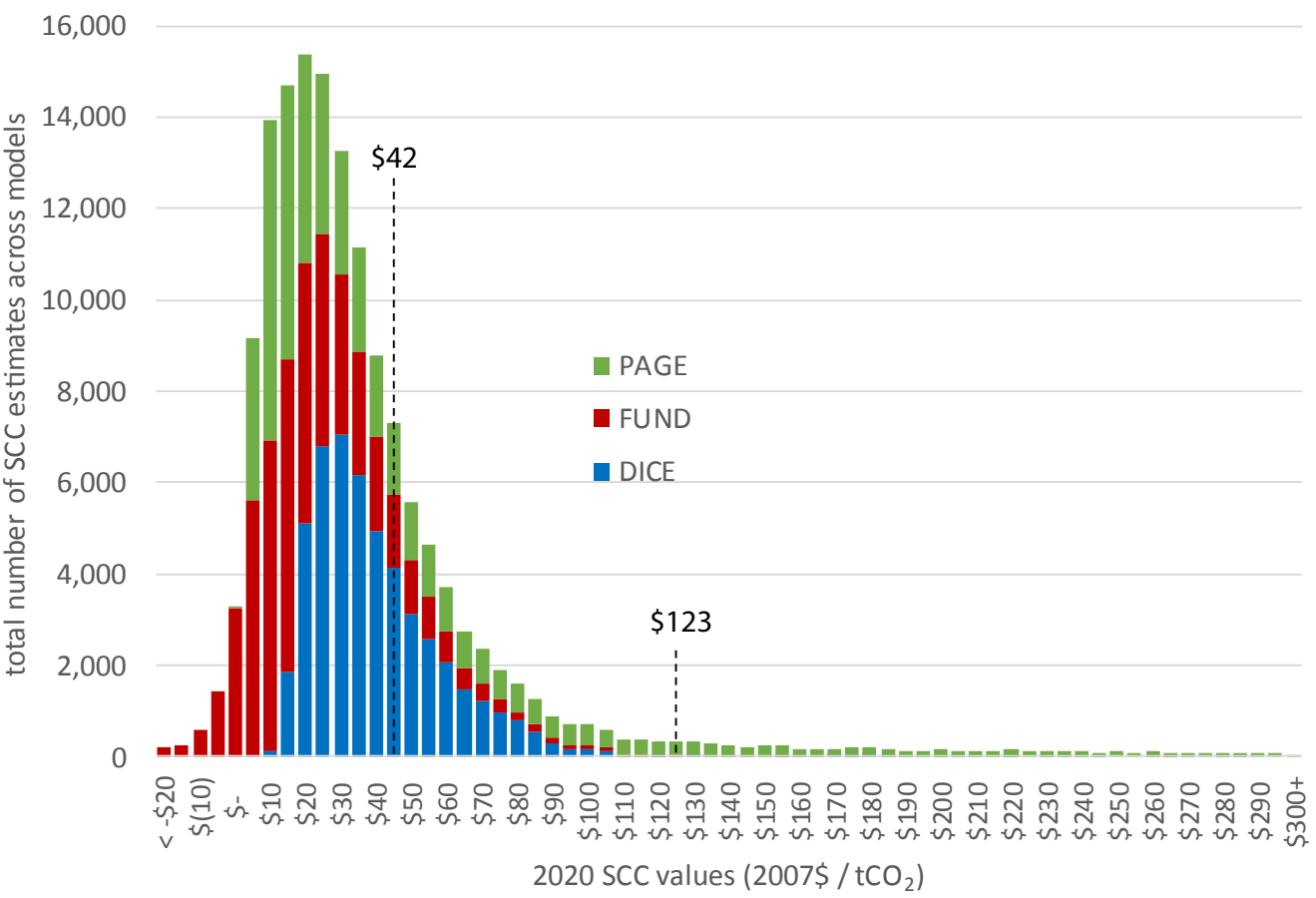

Notes: The figure combines the 50,000 $20203 \%$ discount rate estimates from each of the three USG models to illustrate their influence on the aggregate histogram that determines the official USG SCCs for 2020 at 3\% — the average $(\$ 42)$ and 95 th percentile $(\$ 123)$.

Figure 1. Histogram of the 150,000 USG SCC estimates for 2020 with a $3 \%$ discount rate with estimates from the individual models identified.

A USG estimate is the result of significant aggregation: aggregation within a model - over time, world regions, damage categories, and uncertain inputs and parameters - and aggregation across models. This aggregation obscures the underlying details and drivers of results within models, as well as variation and inconsistencies across models. For example, decomposing the 150,000 SCC results for a given discount rate and emissions year by model (Fig. 1), we immediately observe significant differences in the role each model is playing in the official USG SCC values (the 3\% discount rate average and 95th percentile values of $\$ 42$ and $\$ 123 / \mathrm{tCO}_{2}$ ). ${ }^{2}$ Estimates from FUND alone represent the left tail of the distribution, while PAGE's estimates define the long right tail, and DICE generates a more compact SCC distribution with no negative values and a right tail that contributes to a higher average.

Figure 1 provides a necessary, but insufficient, first step - a first order decomposition of the role of the models in the USG aggregation. To truly understand and assess the results, we need to know what is driving each model's distribution. Despite

\footnotetext{
${ }^{2}$ Figure 1 developed from USG data available at https://www.whitehouse.gov/omb/oira/social-cost-of-carbon. Also see USG Interagency Working Group on Social Cost of Carbon $(2015,2016)$.
} 
the increasingly widespread use of the USG SCC estimates in policy, little is known or available regarding these details and intermediate results, making the values difficult to interpret and evaluate in terms of implied climate risks to society - types and locations over time. Moreover, no study to date has undertaken a direct comparison of the modeling underlying the SCC estimates. With SCC estimates based explicitly on this multimodel approach shaping US policy, and that of states and other countries, understanding the modeling and differences, and the opportunities for and implication of aggregating across models, is essential.

This study presents the first in-depth examination, comparison, and assessment of the three models underlying the USG estimates, as well as the overall multimodel approach. We conduct a model diagnostic and inter-comparison exercise with systematic, independent analyses of the major components of the modeling causal chain: socioeconomics and emissions, climate, and damages. We review model code, program and run components, and isolate and evaluate differences within each component with standardized inputs and diagnostic scenarios that reveal component behavior undiscounted and disaggregated. These analyses elucidate model dynamics behind the SCC that are not reported - intermediate and disaggregated variables such as projected climate and damages over time, regions and categories, as well as characterizations of sensitivity. We generate and evaluate deterministic and probabilistic results for both reference outcomes and incremental responses. Our component assessment findings allow us to then reflect on the overall USG approach. We therefore conclude with a final assessment of the USG SCC experimental design.

The intent of this analysis is not to assess whether the USG estimates are accurate, nor is it to re-compute the SCC. Instead, the objective is to provide the community of policy-makers, stakeholders, and scientists greater technical clarity on the state-of-theart for SCC and global climate damage estimation. Other issues, such as omitted impact categories and biases (e.g., Howard, 2014; Tol, 2009; IPCC, 2007), USG SCC development process (e.g., US GAO, 2014), equity weighting and low intergenerational discounting (e.g., Johnson and Hope, 2012) are beyond the scope of this study. Our focus is on understanding, evaluating, and improving the modeling currently in place, which is a requisite first step before many of these other issues can be broached. Furthermore, while we are analyzing particular versions of the models used for the most recent USG estimates, our perspectives and insights apply to other SCC modeling, other applications of these models (e.g., social cost of other greenhouse gases), ${ }^{3}$ and discussions of aggregate climate risks and goals. With this analysis, we hope to establish a new common analytical ground for moving forward - improving public and scientific understanding, informing future estimation and use, and identifying climate impacts and climate damages research priorities.

${ }^{3}$ USG Interagency Working Group on Social Cost of Greenhouse Gases (2016). 


\section{Methods}

Our analysis is based on versions of the three models as used by the USG, versions that have been modified in important ways from the standard versions found in the literature. See Rose et al. (2014) for details. We evaluate the socioeconomic and emissions, climate, and damage components of each model separately, reviewing model structure and input assumptions, recoding the component in a consistent programming language, running standardized diagnostic scenarios, and comparing the raw results. We run reference and $\mathrm{CO}_{2}$ pulse experiments to reveal behavior. Incremental pulse responses are relevant because SCCs are an estimate of additional damages from an incremental climate response. Total responses are equally important, as they define the reference conditions for marginal damages and allow for direct comparison to other analyses. Across models, there is variation at each component step that would normally propagate through the causal chain. However, we standardize the inputs for each component assessment to isolate component behavior. We evaluate model behavior from numerous perspectives - total/incremental, deterministic/probabilistic, aggregated/disaggregated, reference and sensitivities, and to 2100 and 2300. Some perspectives are in the main paper, many are in the SM.

For the climate component, we evaluate reference paths for temperature and other intermediate climate variables for the USG experiments high and low emissions and non- $\mathrm{CO}_{2}$ forcing scenarios (Table $\mathrm{S} 3$ ). We then re-evaluate each scenario with a $\mathrm{CO}_{2}$ emissions pulse to calculate the incremental response in each climate variable. We use a standardized incremental emissions pulse of 1 billion metric tons of carbon in 2020. In the USG exercise, the emissions pulse in a given year $t$ was implemented inconsistently by the three models in terms of shock size and how it was introduced over time. For our diagnostics, we standardize the pulse size, but retain the model-specific implementations with respect to time (Table S2 and Fig. S8). ${ }^{4}$ We also run the USG climate components and a more sophisticated model, MAGICC6, with Representative Concentration Pathway (RCP) emissions projections (Meinshausen et al., 2011; van Vuuren et al., 2011). See Table S3 for RCP inputs. For the damage component in all three models, we use as inputs the DICE reference and incremental temperature projection results for both the high and low USG emissions from our climate component analysis, and the corresponding socioeconomic projections (Figs. S13 and S14).

We begin our component analyses running each component with central parameter values to understand the fundamental nature of each model and reveal differences in sensitivity and the shape of responses. To learn about the uncertainty being modeled, we also develop probabilistic versions of the climate and damage components of the models and run them with random draws over their model-specific parametric spaces. For the probabilistic analyses with FUND and PAGE, we sample independently over each model's uncertain climate and damage component parameters with 2500 Latin

\footnotetext{
${ }^{4}$ This is a large pulse (roughly $10 \%$ of current global emissions). The climate response appears to scale proportionally with the pulse size, but the many facets of a climate response to pulse size should be explicitly studied in the future.
} 
Hypercube draws. The USG exercise used 10,000 random samples and Monte Carlo and Latin Hypercube sampling for FUND and PAGE, respectively; we use Latin Hypercube sampling for both models, which allows us to use fewer draws while still representing the full sample space. For comparison, using RCP emissions inputs, we also run MAGICC6 probabilistically, and the USG climate components with both model-specific and ECS uncertainties. ${ }^{5}$

\section{Socioeconomics and Emissions Component}

The marginal damages of emissions today are conditional on assumptions about the future evolution of natural and economic systems, beginning with the assumed future global populations, economies, and emissions over the next three centuries. With such a large scope, uncertainty clearly needs to be considered. Our assessment of this component explores the following questions: (1) What sort of socioeconomic and emissions uncertainty is currently represented in the USG exercise? (2) Is there additional uncertainty to consider? and (3) Are results sensitive to alternative assumptions? In this section, we consider the first two questions through comparison to the literature. The third question is discussed, but not explicitly explored until subsequent component assessments.

In the USG SCC calculations, socioeconomic and emissions uncertainty is represented via five alternative futures - four "baseline" with no assumed future climate policy (USG1-4) and one "policy" future (USG5) described as consistent with stabilizing atmospheric concentrations at $550 \mathrm{ppm} \mathrm{CO}_{2} \mathrm{e}$. Each future consists of a set of projections to 2100 for gross domestic product (GDP), population, fossil and industrial $\mathrm{CO}_{2}$ emissions ( $\mathrm{F} \& \mathrm{I} \mathrm{CO} \mathrm{CO}_{2}$ ), land $\mathrm{CO}_{2}$ emissions, and non- $\mathrm{CO}_{2}$ emissions and/or radiative forcing (USG Interagency Working Group on Social Cost of Carbon, 2010). These are then extended in a very stylized way by the USG to 2300 .

Through comparison to the literature and evaluation of the relationships between variables, we find opportunities for improving consideration of uncertainty. Overall, we find that some socioeconomic and emissions uncertainty are captured. However, it is not comprehensively or rigorously characterized, and some are artificial due to implementation inconsistencies.

Specifically, we find that the five scenarios span some uncertainty, but are narrowly focused on uncertainty in a single variable - global $\mathrm{F} \& \mathrm{I} \mathrm{CO}_{2}$ emissions - from one particular study (Clarke et al., 2009). The four baseline scenarios span the $\mathrm{F} \& \mathrm{I} \mathrm{CO}_{2}$ emissions range in the selected study (Fig. 2). However, the range is broader when one fully considers the scenarios literature (Clarke et al., 2009, 2014), and the policy scenario cannot alone represent uncertainty about policy pathways. ${ }^{6}$ The five scenarios, therefore, are not reflective of the full range of uncertainty about future emissions.

\footnotetext{
${ }^{5}$ We ran MAGICC6 probabilistically from magicc.org using the default set-up (Meinshausen et al., 2009).

${ }^{6}$ The policy scenario has an additional issue in that it is not internally consistent. It was constructed by averaging variables independently from four other scenarios.
} 


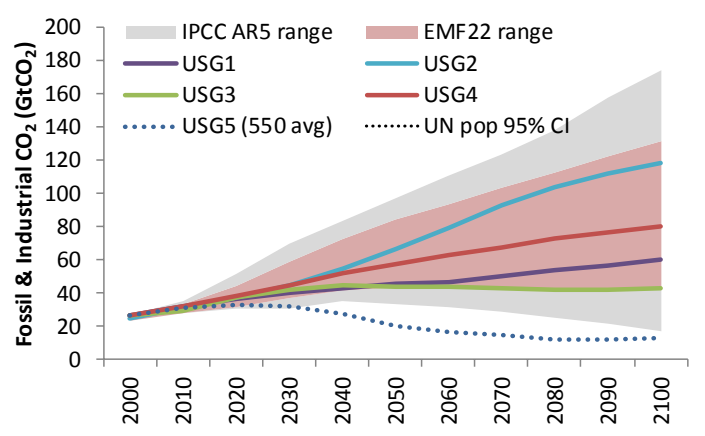

(a)

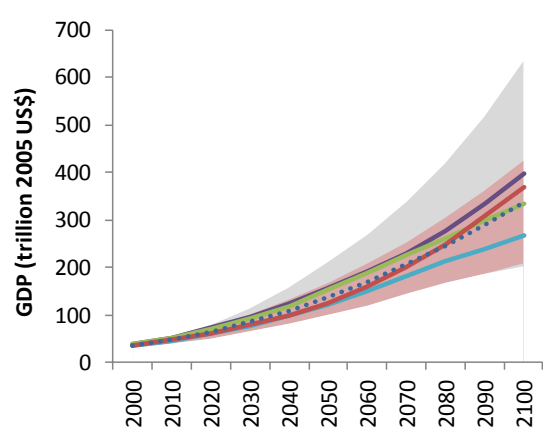

(b)

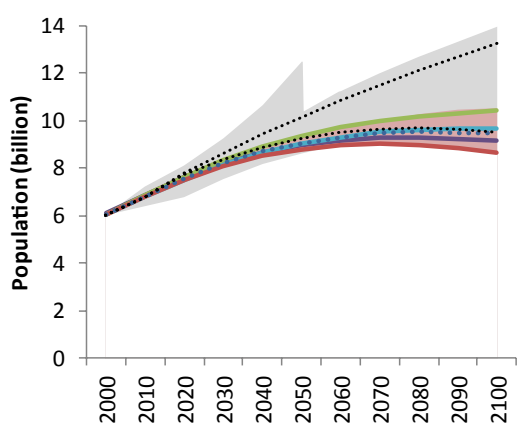

(c)

Notes: Some projections run to 2050. The policy scenario (USG5) is shown for comparison. Literature ranges are from Clarke et al. $(2009,2014)$ and United Nations (2015).

Figure 2. Baseline global fossil and industrial $\mathrm{CO}_{2}$, GDP, and population for USG SCC futures and literature ranges.

Consideration of uncertainty in the other variables is also essential, e.g., Clarke et al. (2014) for GDP and United Nations (2015) for population ("IPCC AR5" and "UN 95\% CI" in Fig. 2 respectively), as is consideration of uncertainty in socioeconomic structure that determines emissions from a future society. For instance, the emissionssocioeconomic relationships in the current projections are arbitrary (e.g., USG2 exhibits high emissions with slow economic growth and USG3 exhibits low emissions with high growth). Uncertainty in socioeconomic structure, such as the energy intensity of economic growth and emissions intensity of that energy, is relevant for projecting emissions, but also for estimating damages, which are sensitive to socioeconomic levels. Finally, weighting of scenarios is important. The USG exercise implicitly assigns $20 \%$ likelihoods to each scenario. No formal process was undertaken to develop probabilities for the five futures. While it is, in general, difficult to assign such probabilities, it is possible to recognize unlikely or less likely futures (such as high emissions for centuries and emissions inconsistent with global trends), and there are methods for developing defensible distributions using historical ranges, parametric uncertainty, and expert 
elicitation. Note that, consistency, uncertainty, and likelihood are also issues for the USG extensions to 2300 (Rose et al., 2014).

The representation of socioeconomic and emissions uncertainty is not only a function of the specification of the inputs, but also how the inputs enter the models. For instance, within the code, we find inconsistency in input implementation that results in differences in what is standardized, exogenous, and even included. For example, DICE and PAGE include more complete sets of non- $\mathrm{CO}_{2}$ radiative forcing constituents than FUND. FUND excludes some elements of forcing, which drive temperature. In addition, the socioeconomic inputs are represented differently in each model. These implementation differences result in artificial differences in projected climate and damages. See Tables S2 and S3 for model implementation details and Rose et al. (2014) for additional discussion.

Overall, our component assessment provides perspectives for the enhanced representation of socioeconomic and emissions uncertainty. Our findings suggest that the USG consideration of uncertainty is incomplete and the distributional specification ad hoc, with additional uncertainty to consider, in both the projected variables currently included and the relationship between variables. With a more comprehensive and rigorous specification, we would expect different posterior distributions of SCC estimates from each model. Unfortunately, it is impossible to know the exact implications for SCC values without developing an improved specification and doing the modeling. Our findings also imply that some of the uncertainty represented in the current USG estimates derives from implementation inconsistency that is not indicative of scientific uncertainty. For example, the specific forcing constituents omitted from the FUND modeling generate an upward bias in FUND's climate projections and SCC estimates. Finally, as shown in the next two sections, each model's climate and damage projections are sensitive to alternative socioeconomic/emissions assumptions, with a more comprehensive consideration of uncertainty likely to have a larger impact on the distribution of SCC results from DICE and PAGE.

\section{Climate Modeling Component}

Each model includes its own reduced-form climate module for translating projected emissions into global mean temperature (GMT) change. Each module estimates $\mathrm{CO}_{2}$ concentrations and radiative forcing, includes equations or assumptions for non- $\mathrm{CO}_{2}$ concentrations and radiative forcing, and derives GMT from total radiative forcing and equilibrium climate sensitivity (ECS).

Our assessment of the climate component explores the questions: (1) How do the climate models underlying SCC calculations behave, and are they similar? (2) What do the incremental climate responses look like from each model, and are they similar? and, (3) How do the USG SCC model responses compare to more detailed climate models? Uncertainty in climate system dynamics is also important and we dedicate a later section to the modeling of climate and damage uncertainty. 
First, from evaluating the code, we observe that the climate module structures and specifications vary substantially across models, as does their implementation in the USG approach (Table S2). As discussed below, we find that both are contributing to differences in projected levels of global warming. For instance, inconsistencies extend beyond the radiative forcings included, noted in the previous section, to climate feedbacks modeled, the relationship of ECS to other parameters, modeling of parametric uncertainty (discussed in uncertainty section), and the implementation of the incremental $\mathrm{CO}_{2}$ pulse.

For our diagnostics, we begin by running the climate components with standardized inputs - the USG high and low emissions projections - to explore reference pathway responses. As shown in Fig. 3 and the SM, we find substantial variation across models in all aspects of projected climate: carbon cycle (Fig. 3(a)), translation of concentrations to forcing and the constituents of forcing (Figs. S4(a,b)-S6(a,b)), and temperature dynamics as a function of forcing (Fig. 3(c)). For example, by 2100, projected GMT results vary by $1^{\circ} \mathrm{C}$ in the higher emissions reference scenario, and by $0.5^{\circ} \mathrm{C}$ in the lower emissions reference. For SCC estimates, this matters, as the models are evaluating marginal damages relative to different reference warming levels.

For the high emissions scenario, DICE and PAGE project significantly greater warming than FUND. However, in deriving projected temperatures, it is PAGE that has the lowest projected $\mathrm{CO}_{2}$ concentrations, $\mathrm{CO}_{2}$ forcing, and total forcing. Behind this shift in ordinal ranking are pronounced differences between models in the translation of total forcing to temperature. In particular, a long temperature lag assumption in FUND results in a more gradual development of temperature, while PAGE's temperature responds more quickly to the ECS parameter than the other models (discussed below), resulting in a large temperature response to forcing. Importantly, some of the variation in results is driven by the differences in model structure and the USG implementation, differences that may be artificial.

From our sensitivity analysis, we gain insights into the responsiveness of the models to uncertain emissions and input assumptions, which helps us to further understand the distribution of SCC estimates from each model. Specifically, our sensitivity analysis reveals FUND to be the least sensitive to alternative projected emissions (Fig. 3(c)), as well as the ECS (Fig. S7). DICE's temperature projections are the most sensitive to alternative emissions projections, as are the DICE projected $\mathrm{CO}_{2}$ and total forcings, while PAGE's temperature projections are significantly more sensitive to alternative ECS values than the other models.

ECS is a key uncertain parameter, and there are differences in how ECS affects the temperature transition in each model. To ensure consistency with historical temperature observations, an increase in ECS should be accompanied by both faster ocean heat uptake (or in simple models, a slower response rate of average surface temperature) and larger negative aerosol forcing (e.g., Urban et al., 2014). DICE and FUND adjust the temperature response rate, and DICE further includes a moderating ocean feedback. PAGE, however, does not include any countervailing adjustments, and consequently its temperature results are significantly more sensitive than those from the 


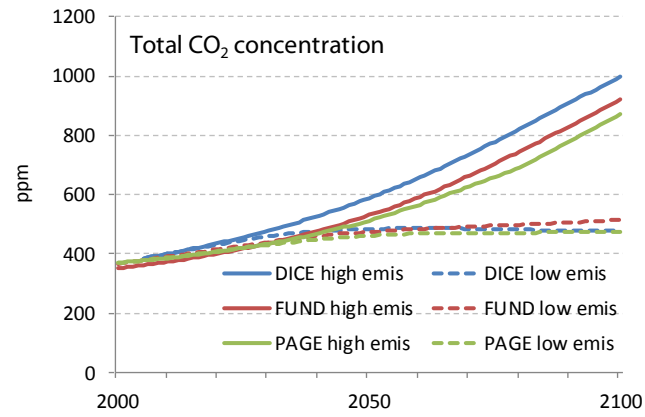

(a)

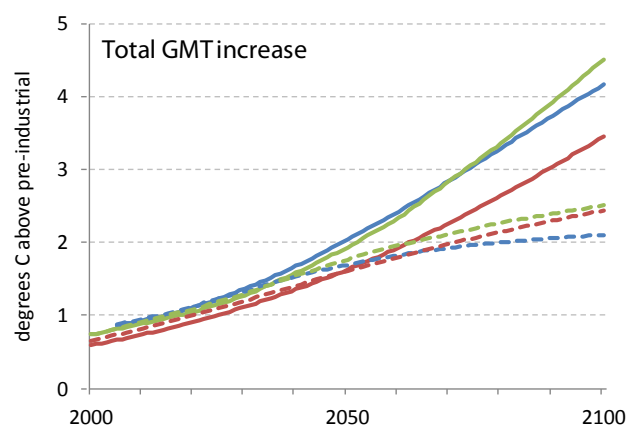

(c)

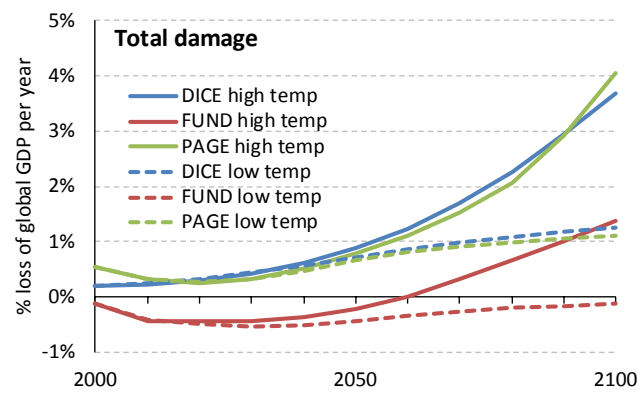

(e)

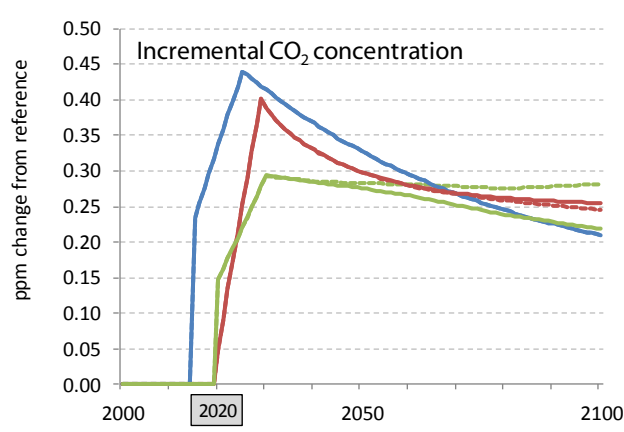

(b)

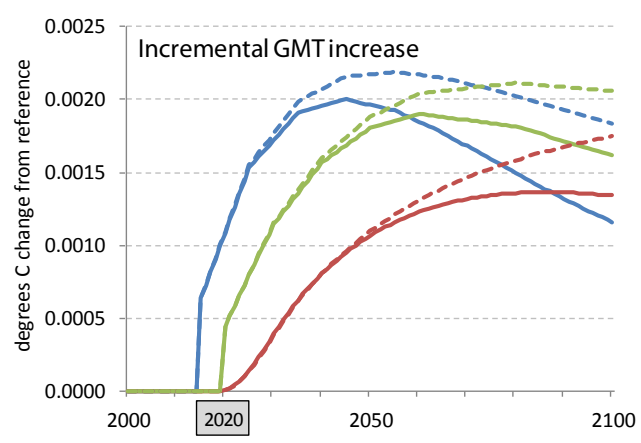

(d)

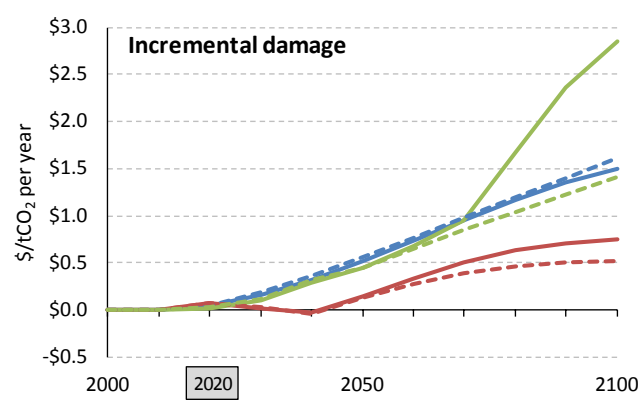

(f)

Figure 3. ((a), (b)) Total and incremental responses in $\mathrm{CO}_{2}$ concentrations and ((c), (d)) GMT increase with standardized emissions inputs, and ((e), (f)) global damage responses with standardized temperature and socioeconomic inputs. See Figs. S2, S3, S9, S10, S17, and S22 for results through 2300 .

other models (Fig. S7). Note that, none of the models currently adjust aerosol forcing for alternative ECS values.

Next, we evaluate incremental responses with our $2020 \mathrm{CO}_{2}$ pulse experiment (Methods). In general, we find incremental GMT responses that peak and decline with atmospheric decay of the pulse from all the models, but with significantly different 
timing, levels, and rates entering into damage and SCC calculations (Figs. 3(d) and S10 for results to 2300). Levels diverge by as much as a factor of two in 2050, with DICE producing an earlier and higher peak, followed by PAGE, and both well above the incremental GMT response from FUND. In Fig. 3(d), we also observe that all the models exhibit higher incremental temperature responses from scenarios with lower emissions due to the concavity of the logarithmic function for $\mathrm{CO}_{2}$ forcing (see Fig. S11(a,b) for forcing responses).

The differences in the incremental pathways derive from differences in component structure, as well as pulse implementation. Inconsistency in the implementation of the pulse between models, with variation in how the pulse is spread over years (Fig. S8), results in DICE and PAGE producing incremental climate effects in 2020 higher than they would be otherwise (Figs. 3(b) and 3(d)).

We also observe carbon cycle response differences due to the treatment of feedbacks to terrestrial and ocean carbon uptake. DICE includes no feedbacks, making its incremental $\mathrm{CO}_{2}$ concentration response insensitive to the emissions scenario, while FUND and PAGE include feedbacks, but with responses that exhibit different signs and absolute magnitudes (Figs. 3(b) and S9 for results to 2300). PAGE's slower carbon cycle is also evident in a significantly lower $\mathrm{CO}_{2}$ concentration spike that is ultimately offset by the strength of its conversion of forcing to temperature. Meanwhile, FUND's higher concentration spike is offset by its slow temperature response. Finally, the sensitivity of incremental GMT responses to emissions and ECS assumptions is consistent with what we observed for total GMT.

For comparison, we conducted the same pulse experiment in MAGICC, a more sophisticated climate model designed to emulate complex earth system models. Using two alternative carbon cycle representations, we find similarities in the reference temperature projections between MAGICC and the USG models, but also notable differences in initial levels and rates of change (Fig. S12). Most relevant to the SCC, we find substantial differences in the incremental climate response (Fig. 4). In MAGICC, atmospheric decay begins immediately after the pulse, leading to a much earlier peak in incremental temperature than in the USG models (especially FUND), and the decline following the peak is slower, in particular when emulating models with stronger temperature feedbacks. Additional evaluation is merited, but the MAGICC pattern for the incremental response appears to be more consistent with complex models (Joos et al., 2013).

From our component assessment, we conclude that the modeling and implementation of the climate system is very different across models. These are differences that affect results and need justification, with some not indicative of structural uncertainty (e.g., pulse implementation, carbon cycle feedback, forcing constituents). These differences impact projected incremental temperature responses and ultimately SCC estimates. FUND's more modest and less sensitive temperature responses are contributing to lower SCCs and a more compact SCC distribution; PAGE's higher, earlier, and more sensitive temperature responses contribute to higher SCCs and a wider range 


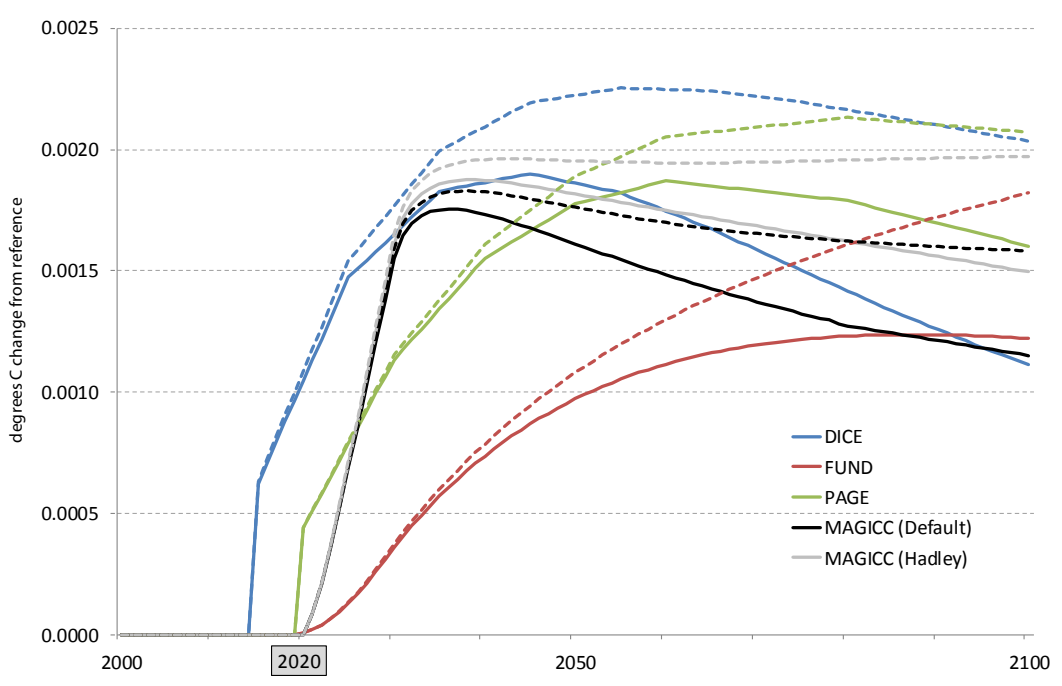

Figure 4. Incremental responses in GMT to 2100 for MAGICC versus the USG models with standardized RCP emissions inputs (RCP8.5 solid, RCP3-PD dashed).

of temperature projections and SCC values, including PAGE's prominence in the right tail of the SCC distribution (Fig. 1); and, DICE's higher and earlier temperature responses contribute to higher SCCs, but without a climate feedback, DICE's SCC distribution is more compact ceteris paribus. Finally, we note that the higher incremental temperature response off a lower emissions pathway contributes to a higher SCC, though it is a combination of factors, including damages, that determine whether the resulting SCC is higher or lower.

\section{Climate Damages Modeling Component}

These three IAMs are among the few available models for monetary valuation of global climate damages. As an aggregate metric, an SCC is not particularly intuitive or easily interpreted: it encapsulates damages projected to occur at different points in time, in different regions of the world, and for different types of impacts. The official USG SCCs are not only an aggregation of damages within models, but also across models and scenarios. Understanding, evaluating, and comparing SCC estimates requires the raw undiscounted and disaggregated damage outcomes over time from the individual models, and alternative model runs. These intermediate results, however, have not been made available until now. Our assessment of the damage component fills this gap and explores the following questions: (1) What are the detailed constituents of damages underlying SCC calculations? (2) How sensitive are the damage estimates to alternative assumptions and formulations? and (3) How do damage estimates respond incrementally to a marginal change in emissions?

We begin by evaluating the damage component formulation of the models. Each model estimates global damages by calculating some degree of disaggregated 
damages, with substantial variation across models in structure, specification, and implementation (Table S2). For example, DICE is globally aggregated with two categories of damages as a fraction of GDP increasing quadratically with GMT and sea level rise (SLR), respectively; while, FUND derives damages for 16 regions and 14 damage categories that respond to a broader set of drivers such as regional temperatures, temperature rate of change, $\mathrm{CO}_{2}$ concentrations, population, income, and technological change; and, PAGE estimates damages for eight regions and four categories, including a generic discontinuity impact, with more drivers than DICE but fewer than FUND. While global damage results can be readily compared between the models, the differences in the region-category resolution of damages limit us to a few sub-global comparisons. It is worth noting that, even a highly aggregate damage structure, through its calibration, has implied patterns of damages in terms of damage locations and types for different levels of warming. This information, however, is not discernable from current documentation. ${ }^{7}$ Going forward, these kinds of details are critical for transparency and evaluation.

Compiling and evaluating the literature basis for the damage specifications (Table S4), we find the models relying directly and indirectly on older studies that do not reflect current knowledge of climate impacts (e.g., Field et al., 2014). This is, in part, because the more recent literature is not readily usable for this application. Moreover, we find interdependencies among the models, with DICE and PAGE specifications based on damage estimates from previous DICE, FUND, and PAGE results. This issue is discussed later.

For our diagnostics, we begin by running the damage components with standardized high and low GMT projections and socioeconomic inputs (Methods). Despite the major structural differences in damage components, DICE and PAGE project very similar global annual damages over the century, both substantially higher than those from FUND, which projects net benefits for much, or all, of the next century (Fig. 3(e)). By 2100, the models differ in annual damages by as much as a factor of three, with differences growing beyond 2100 due to more rapid growth in damages from DICE and PAGE (Fig. S17). Numerous factors contribute to the very different perspectives on the potential damages for a given climate and society, including intermediate projections, functional forms, individual damage categories, and other model idiosyncrasies. For instance, we find that for the same GMT projection, intermediate outputs of SLR and regional temperatures vary significantly, with FUND projecting up to twice the SLR by 2100 as DICE and PAGE, and PAGE's temperature pattern-scaling producing notably warmer regions than FUND's (Figs. S15 and S16(a,b)). Below, we disaggregate damages and discuss the contributions of other factors.

\footnotetext{
${ }^{7}$ For instance, National Academies of Sciences, Engineering, and Medicine (2017) noted that DICE damages at the global level have been updated and calibrated to meta analyses, but the assumed region and category structure of damages are still based on earlier work by Nordhaus and Boyer (2000).
} 
The slopes of the implied damage functions of temperature are particularly relevant for the SCC, as they indicate the marginal damage response to incremental temperature change. We tease these out by scaling our GMT input projection. We find that across categories, regions, and models, damages differ in responsiveness to warming, including differences in sign (damage versus benefit). Figure 5 presents damages by category and region as a function of temperature with a fixed 2050 society. Overall, the models provide very different damage pictures in terms of potential benefits and costs, damage locations, and rate at which damages might increase with warming. For DICE, non-SLR damages accumulate most quickly (quadratically) with warming. For FUND, the dominant damage responses (positive and negative) are increased cooling costs, avoided heating costs, agriculture net benefits up to $5^{\circ} \mathrm{C}$ warming, and water resource damages. For PAGE, SLR and noneconomic damages are the most responsive at lower levels of warming, while noneconomic and economic damages are the most responsive at higher warming levels, with the expected damage from the discontinuity rising steeply beyond $3^{\circ} \mathrm{C}$ of warming. Interestingly, the cost of adaptation in PAGE is constant, small, and unresponsive to temperature. Note that damages that accumulate over time (e.g., SLR) are not captured by these experiments (more below).

The different model aggregations make direct comparison across models challenging. SLR damage is the only damage category present in all three models. In this case, FUND projects the least amount of SLR damage, despite projecting more SLR for a given temperature. Aggregating FUND and PAGE damage categories into harmonized economic and noneconomic groupings, we find that PAGE's economic and noneconomic damages dwarf FUND's, with the economic damages sometimes differing in sign (Fig. S19).

Regarding regional damages, the responses are fundamentally different in FUND and PAGE (Fig. 5). FUND projects net benefits for a few regions of the world, even up to $4.5^{\circ} \mathrm{C}$ GMT rise, while PAGE projects net damages for all regions and levels of warming. Developing regions dominate FUND's damages, while developed regions dominate PAGE's, especially at lower temperatures, and significantly exceed the developed region damages from FUND (Fig. S20(a)). In FUND, China dominates the overall response, switching from net benefits to net damages around $3^{\circ} \mathrm{C}$, driven by the country's damage coefficients, elasticity parameters, and income and population growth. Note that regional damage responses in PAGE grow proportionally by construction, as regional damages (SLR and non-SLR) are scaled off EU damages based on relative coastline, with a small adjustment for per capita income (Hope, 2011). FUND, on the other hand, calibrates regional damage functions that respond uniquely to warming.

From additional sensitivity experiments, we find that damages are responsive to income, as well as temperature (Fig. 6), and that some models are systematically more or less sensitive. Damages increase with temperature and income in all the models; however, DICE and PAGE damages increase most quickly, with PAGE the most responsive to both drivers, and FUND the least responsive to both. Globally, damages are relatively unresponsive to population size, with FUND and PAGE 

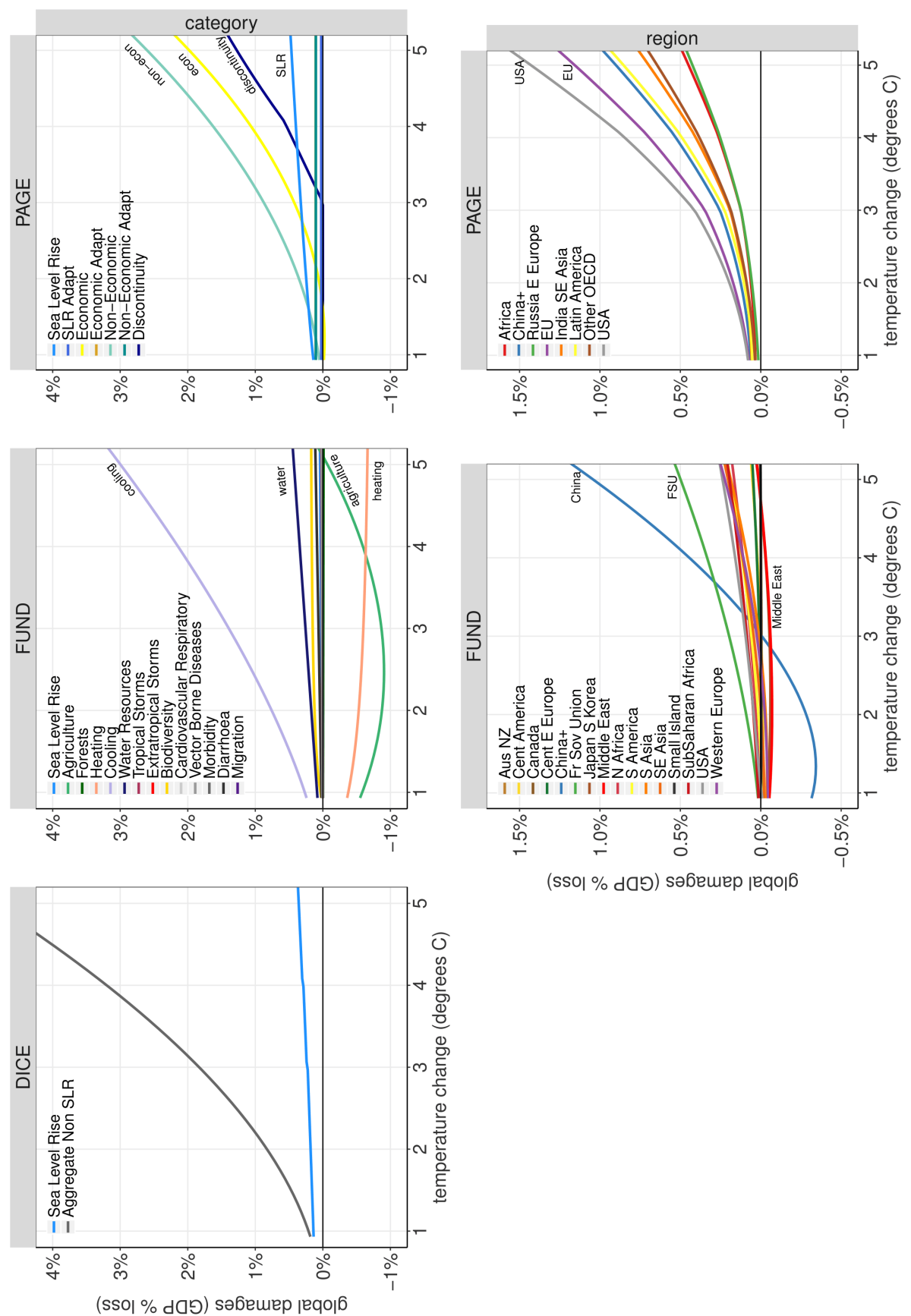

(ssol \% dav) sə6emep ןeqo|6 

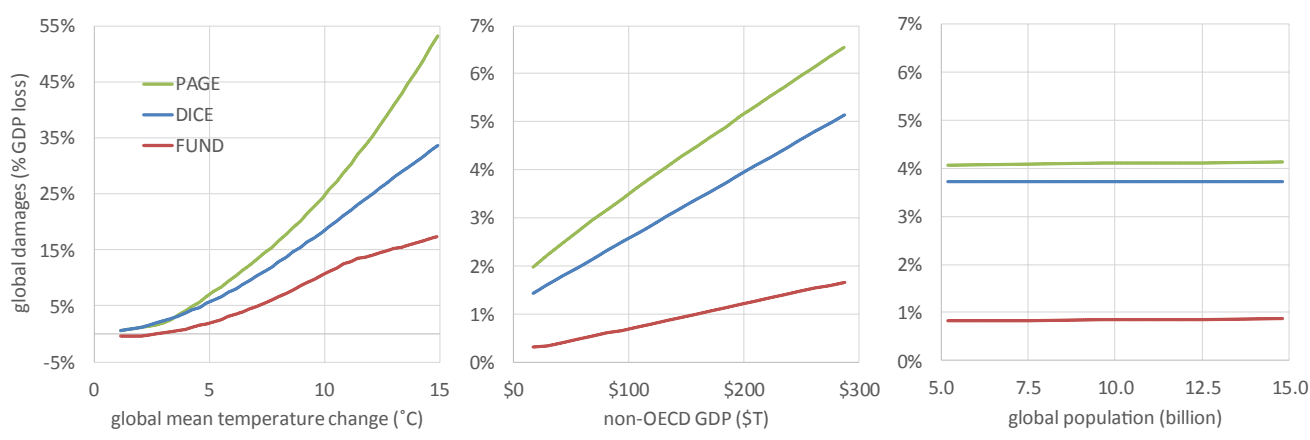

Notes: For each chart, only a single driver is varied from the standardized USG2 conditions in 2100 (2100 values: global GDP $\$ 268$ trillion, non-OECD GDP $\$ 162$ trillion, global population 9.6 billion, GMT above preindustrial $4^{\circ} \mathrm{C}$ ).

Figure 6. Implied global damage functions with respect to GMT change, non-OECD income, and global population ( $y$-axis' ranges vary).

exhibiting only slight positive correlations. This is, in part, because of how population enters each model, as well as smaller projected relative damages for categories with more direct population ties (e.g., health). ${ }^{8}$ In all models, the absolute value of damages increases with income simply because there is more to affect (i.e., exposed society); however, FUND projects increasing net benefits when there are low levels of warming, and damages respond differently to income in rich versus poor countries, moderated by regional dynamic vulnerability (i.e., resiliency as a function of per capita income). See Figs. S18 and S21(a,b) for additional results.

Next, we examine incremental damages, the additional damages from a standardized pulsed temperature pathway versus a reference (Methods). We find that, despite a decaying incremental temperature input, annual incremental damages increase over time in all models due to income growth and rising reference temperatures (Figs. 3(f) and S22 for results to 2300). The differences in incremental annual damages are a reflection of the different damage gradients we observed above. Rising temperatures and incomes result in larger marginal increases in damages in DICE and PAGE. In addition, PAGE's incremental damages rise sharply with a marginal increase in expected discontinuity damages. Meanwhile, incremental annual damage growth declines notably over time in FUND as income growth reduces vulnerability.

To highlight prominent features in the pattern of incremental damages over time in each model, we develop a custom region-category disaggregation of incremental annual damages (Fig. 7). In DICE, incremental non-SLR damages dominate through 2100, but incremental SLR damages, which accumulate over time, become the primary

\footnotetext{
${ }^{8}$ Our population sensitivity analysis scales global population growth with the regional distribution fixed. Future analysis should consider sensitivities on the distribution of growth. In DICE, population enters as an input to total factor productivity, income, and the capital stock. In FUND, population defines per capita income and is an explicit input into the following damage categories: water resources, energy consumption, ecosystems, human health damage categories, and tropical storms. In PAGE, population defines per capita income, which is an input into each of the damage categories in the model.
} 


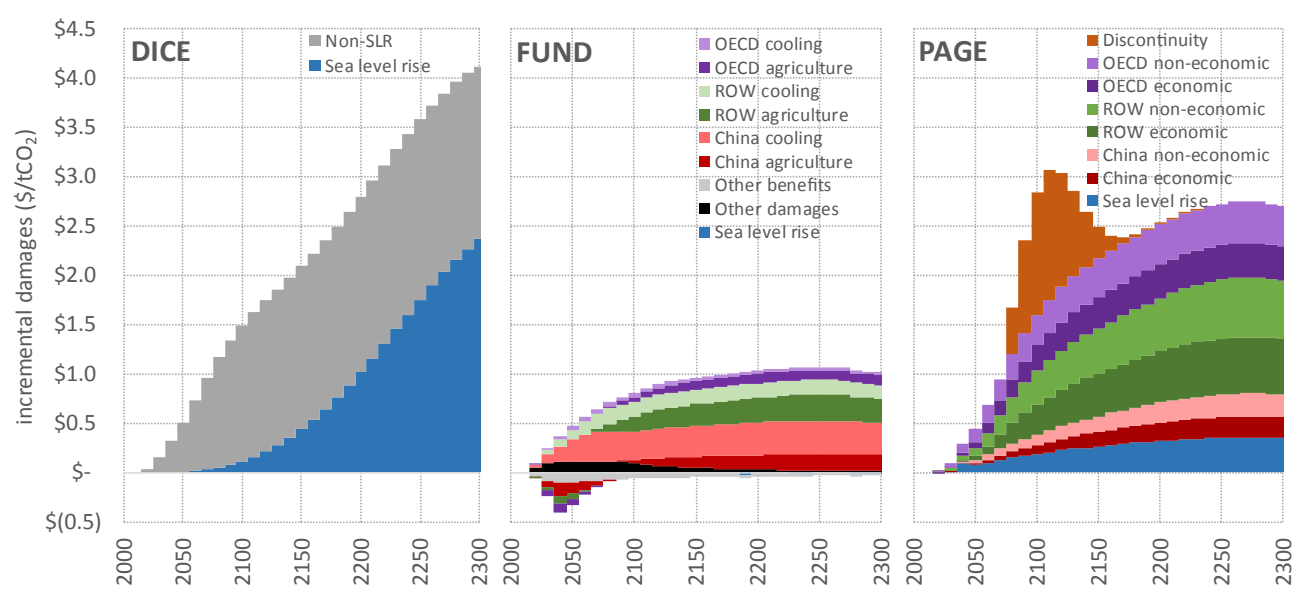

Figure 7. Key factors of annual incremental global climate damages to 2300 with the standardized high temperature and USG2 socioeconomic inputs.

annual damage by 2300. Yet in FUND, incremental SLR damages are almost nonexistent, while incremental cooling (damages) and agriculture (benefits then damages) dominate, with China accounting for approximately half of cumulative incremental damages. In PAGE, incremental noneconomic and economic damages are more consequential than SLR (with its sublinear functional form), and incremental discontinuity damage with its $3^{\circ} \mathrm{C}$ GMT threshold arrives slightly earlier due to the additional $\mathrm{CO}_{2}$ from the emissions pulse. Incremental damages in developed countries (OECD) dominate PAGE's damages through 2050, but are eventually surpassed by annual developing country damages. To 2100 , roughly half of PAGE's incremental damages occur in developing countries, compared to $80 \%$ in FUND, with incremental damages in China much larger in FUND than in PAGE. Cumulatively through 2300 (Fig. S20 (b)), the leading incremental damage category-region combinations in FUND are cooling in China (33\% of incremental damages), followed by agriculture in China and South Asia $(9 \%, 6 \%)$. In PAGE, they are noneconomic damages in Latin America, China, and the US $(8 \%, 7 \%, 6 \%)$, followed by economic damages in Latin America, China, and India (all 6\%).

From this component assessment, we conclude that damage component modeling varies substantial across models with key features contributing to higher or lower SCC values and different SCC distributions. For instance, damages in FUND are less responsive than in the other models to two primary drivers of damages - temperature and income - due in part to lower regional temperatures, agriculture and heating benefits, and adaptation responses. These elements contribute to lower SCCs and tighter SCC distributions. Damages in DICE and PAGE, on the other hand, are more responsive to the drivers due in part to assumed net damages for all levels of warming, income, and population, higher regional temperatures (PAGE), explicit or implicit functional forms with higher exponents, less prominent adaptation, unspecified 
discontinuity damages (PAGE), and regional scaling of damages (PAGE). These elements contribute to higher SCCs and broader SCC distributions.

\section{Model-Specific Uncertainty}

Results to this point in our study reflect calculations based on the models' central parameter values and sensitivity analysis with inputs and the ECS parameter. Recall, however, that the models were run probabilistically in the USG exercise. Our review of the probabilistic specifications of the models finds that parametric uncertainty is handled quite differently across models. Specifically, the USG experimental design specifies standardized ECS uncertainty for all models, but the FUND and PAGE climate and damage components are also run with their native parametric uncertainty. For FUND, there are 11 and over 400 independent general and regional uncertain parameters in the climate and damages components, respectively, for PAGE, 10 and 35, respectively, and for DICE, none. The specific uncertain parameters in FUND and PAGE differ, as does the assumed distributional specifications, with triangular distributions assumed for all PAGE parameters, and various distributional forms assumed in FUND (see Table S2 for details on the different types and specifications of parametric uncertainty). As discussed in Methods, we develop and run probabilistic versions of the climate and damage model components to learn about the uncertainty modeled.

In the climate component, we find substantially larger climate uncertainty and higher central tendencies being represented and projected through 2300 in PAGE than in FUND (Fig. 8). Indeed, the $90 \%$ confidence interval, 5th to 95th percentiles, for incremental GMT change in 2050 from PAGE is 30 times wider than FUND's, with PAGE producing higher annual mean increases in incremental temperature. Re-running the USG climate components with RCP inputs and model-specific and ECS uncertainties, we can compare to probabilistic results from MAGICC. MAGICC has an alternative characterization of parametric uncertainty with 82 uncertain parameters (Meinshausen et al., 2009). Comparing to the resulting MAGICC temperature distribution for the high emissions scenario (RCP8.5), we find FUND suggesting significantly less uncertainty and risk, and PAGE significantly more (Fig. 9). Interestingly, the DICE distribution is the most closely aligned with MAGICC, however the DICE distribution represents only ECS uncertainty. Comparing results for the low emissions scenario (RCP3-PD), we find that all the USG models suggest very different temporal profiles and significantly more uncertainty than MAGICC. Note that in Fig. 9, we are comparing 66\% confidence intervals, which is all that was available for the online version of MAGICC. The tails are clearly important to SCC calculations and should be further explored.

In the damage component, we find FUND representing and projecting more uncertainty than PAGE in annual incremental damages through 2100, and less beyond (Fig. 8). The broader first century distribution in FUND is driven by uncertainty in cooling, agriculture, and water resource damages in China, South Asia, and South America, while the primary uncertainty drivers in PAGE are noneconomic and 


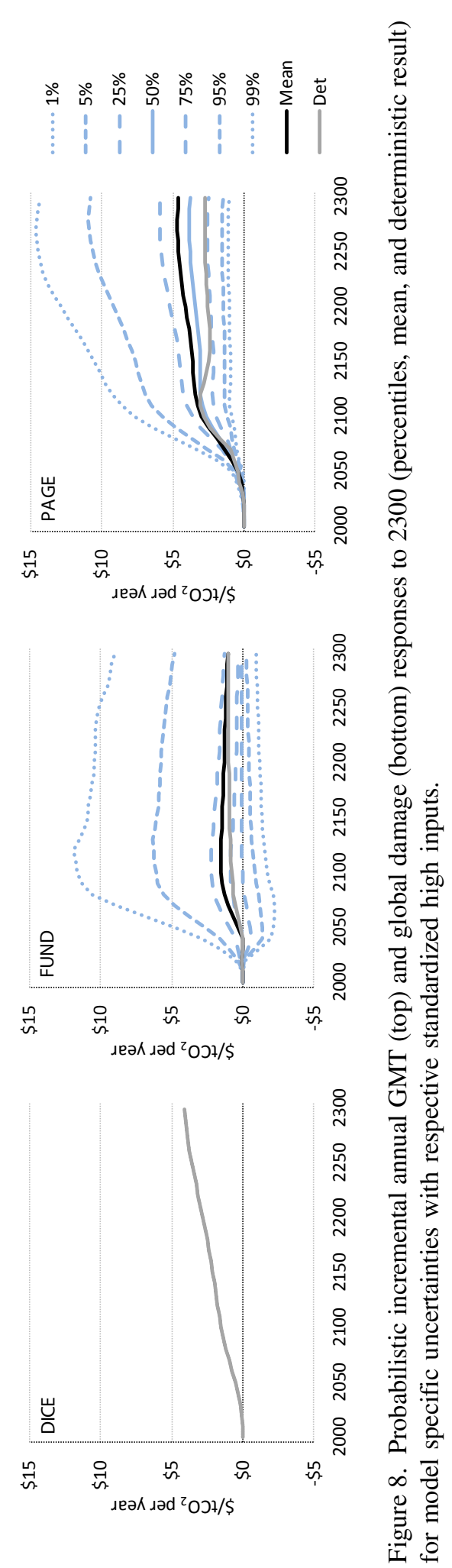



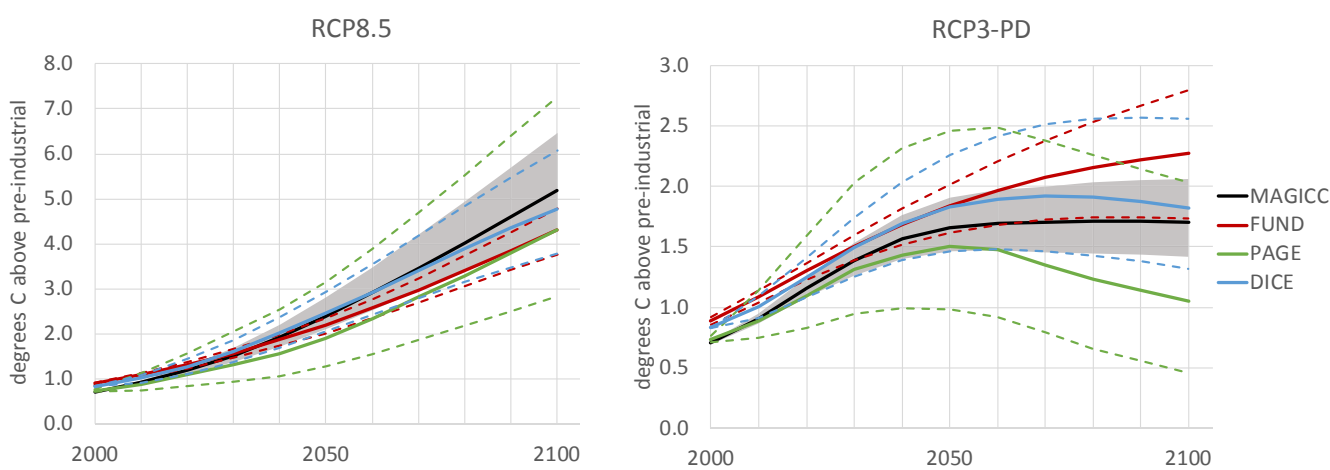

Figure 9. Probabilistic GMT to 2100 for MAGICC versus the USG models with standardized RCP emissions inputs (solid = median, dashed = USG model 17th-83rd percentile, shaded $=$ MAGICC 17 th-83rd percentile. $y$-axis' vary.)

economic damage in China, Latin America, and India (Rose et al., 2014). We note that some FUND and PAGE parameter combinations lead to annual damages equal to or greater than $100 \%$ of the economy for some regions at higher levels of warming, which can mean a regional annual incremental damage effect of zero. ${ }^{9}$ Whether the magnitudes of reference losses projected by the models make sense is a topic onto its own and beyond the scope of this study. Throughout the time horizon, PAGE produces higher annual mean incremental damages, a point germane because the USG aggregation approach emphasizes means. There are, unfortunately, few examples in the literature of uncertainty distributions over climate damages, for a given climate and society, to which the FUND and PAGE ranges can be benchmarked. A systematic study of damage uncertainty based on the current impacts literature is a key research need.

From our assessment of model specific uncertainties, we conclude that there is inconsistency across models in the representation of parametric uncertainty. Furthermore, the PAGE formulations yield both higher average incremental temperatures and damages. From Fig. 8, we also note that the average results differ from the deterministic results, with similar to slightly lower average annual incremental temperatures and clearly larger average annual incremental damages. However, average incremental temperature projections can exceed deterministic outcomes when we include ECS uncertainty, with the effect strongest in PAGE. These findings, in addition to the tendencies identified earlier from our deterministic experiments, contribute to wider PAGE SCC distributions with larger right tails, and higher average estimates.

\footnotetext{
${ }^{9}$ FUND enforces a $100 \%$ maximum on total economic damages, but not on the sum of economic and noneconomic damages. PAGE has separate saturation approaches for economic and noneconomic damages that does not explicitly limit total damages to less than $100 \%$ of the economy. One justification given for greater than $100 \%$ losses is that noneconomic damages can push total damages above income levels (Anthoff and Tol, 2013). In this case, noneconomic damages should not be interpreted as willingness to pay estimates, which are budget constrained.
} 


\section{Summary of Component Assessment}

The component assessments above have identified differences in model structure and behavior, and revealed model tendencies that help us interpret SCC results. Our independent component assessments have isolated and elucidated differences across models in intermediate projections, sensitivity, and implementations - socioeconomics, emissions, $\mathrm{CO}_{2}$ concentrations, radiative forcing, temperature, regional temperatures, SLR, and the magnitude and composition of damages. We found that some differences are artificial, and all the differences need justification. Most importantly, we found tendencies in intermediate results due to component modeling elements that contribute to differences in the observed SCC outcomes between models. For instance, we found FUND producing the lowest incremental temperature and damage responses, and having more muted sensitivity than the other two models to uncertainties about emissions, ECS, and temperature, as well as responses to income that contribute to a lower average SCC. DICE and PAGE, on the other hand, generated higher and earlier incremental temperature and damage responses, with DICE the most sensitivity to emissions, while PAGE is the most sensitive to ECS and temperature, as well as income at higher levels of warming, and PAGE's parametric uncertainty further contributing to higher incremental temperatures and damages. Together, PAGE's responses combine to yield SCC distributions with longer and fatter right tails and higher averages than the other models. DICE's and FUND's distributions are more compact, and FUND, with the lowest averages, is the only model producing distributions that include the possibility of global net benefits.

\section{USG Experimental Design and Discussion}

Overall, our component assessments have allowed us to trace SCC differences back to component modeling, and even specific features, which accommodate the evaluation of individual model SCCs in terms of concrete underlying elements. The assessments have also provided comparable details about the models and modeling that allow us to reflect on the overall USG experimental design. To date, SCC estimates in the literature have only been generated by individual models with nonstandardized uncertainties (e.g., Tol, 2009). The USG experimental design, however, is novel - a multimodel, probabilistic approach with standardized and model-specific uncertainties, and an aggregation procedure. The USG experimental design is defined by a set of methodological choices, which we have itemized in Table 1.

Each of these choices can affect results (examples below), and as choices, there are alternatives. It is therefore important that the choices be clearly communicated and scientifically supported for peer and public evaluation. Without such information, it is difficult to interpret results and evaluate the approach. The kind of transparency our study is providing is an example of what would be useful, along with justification. The conceptual motivation behind many of the above choices is pragmatic. For instance, given the geographic and temporal scope of the modeling, uncertainty should be 
Table 1. USG experimental design features and choices.

\begin{tabular}{|c|c|}
\hline Experimental design feature & USG choices \\
\hline Model & $\begin{array}{l}\text { - Use multiple models } \\
\text { - Use DICE, FUND, and PAGE } \\
\text { - Modify models from native formulations }\end{array}$ \\
\hline $\begin{array}{l}\text { Projected socioeconomics and } \\
\text { emissions/forcing }\end{array}$ & $\begin{array}{l}\text { - Use partially standardized exogenous alternative socioeconomic and } \\
\text { emissions/forcing projection inputs } \\
\text { - Use five projection sets based on Clarke et al. (2009) } \\
\text { - Extrapolate each projection variable from } 2100 \text { to } 2300\end{array}$ \\
\hline ECS parameter & $\begin{array}{l}\text { - Use a standardized ECS parameter value distribution and choose a random } \\
\text { sampling procedure }\end{array}$ \\
\hline Other input parameters & $\begin{array}{l}\text { - Use model specific uncertainty distributions, make assumptions about } \\
\text { correlations, and choose a random sampling procedure for various other } \\
\text { FUND and PAGE climate and damage component parameters }\end{array}$ \\
\hline Discounting & $\begin{array}{l}\text { - Use constant discounting } \\
\text { - Use three alternative discount rates } \\
\text { - Use } 2.5 \%, 3 \% \text {, and } 5 \%\end{array}$ \\
\hline Model runs and results & $\begin{array}{l}\text { For each official USG SCC.. } \\
\text { - Run each model } 50,000 \text { times (with 10,000 random parameter draws for } \\
\text { each socioeconomic/emissions projection) } \\
\text { - Aggregate results across models into overall distributions by discount rate } \\
\text { with equal weighting of models and socioeconomic/emissions projections } \\
\text { - Select specific values from the overall distributions (averages for each } \\
\text { discount rate, and one 95th percentile) }\end{array}$ \\
\hline
\end{tabular}

embraced and incorporated. Considering multiple models, uncertainty in socioeconomic futures, and uncertainty in climate dynamics and damage specifications are examples of doing so. Discounting is also appropriate for aggregating effects over time.

Our component assessments provide us with an intimate understanding of the modeling that allows us to reflect on the overall experimental design and identify opportunities for improvement. Using multiple models is a means for accounting for differences in expert opinion about the structure and dynamics of social, economic, and physical processes. Upfront consideration of different models is prudent, but it also creates challenges regarding transparency, justification, comparability, and independence that need to be considered in deciding whether to select one model, multiple models with a weighting scheme, or to develop a new model.

A multimodel approach in which results are averaged across models and assumptions (with an implicit equal weighting) should be used when each model is generating comparable and unique information. We find, however, fundamental structural, parametric, and implementation differences across the models, in all components, that 
are artificial rather than a reflection of differences in expert opinion or scientific uncertainty about an element. In addition, the models have significant dependencies, especially in the representation of damages. Review, and possibly modification, of component specification and implementation differences and dependency is an essential future activity for more comparable estimates, which might also provide a model weighting scheme rationale. Such an assessment should, among other things, include evaluation and justification of modeling differences in emissions and radiative forcing, carbon cycles, ECS implementation, climate feedbacks, pulse implementation, empirical bases for damage responses, and parametric uncertainty implementation.

In addition to improving the representation of structural uncertainty, our component assessments identify opportunities for improving the representation of other types of uncertainty. First, the current USG standardized uncertainties could be revised to capture the uncertainty available in the current literature for socioeconomics, emissions, and ECS. There are legitimate alternatives, as well as constraints on what is reasonable and comparable, to consider. Regarding ECS, see Bindoff et al. (2013) for updated ECS distribution assumptions, which includes alternative distributions that would allow for explicitly incorporating uncertainty about the distribution itself. Second, there are additional uncertainties that could be included (e.g., socioeconomic structure, 2300 extrapolations, climate modeling specifications). Finally, parametric uncertainty should be clearly characterized and considered across all models to the extent possible.

Our assessments also suggest that the USG estimates could be made more robust (insensitive to alternatives) to provide greater confidence in final SCC estimates. Our component analyses have shown the climate and damage outcomes to be sensitive to, among other things, emissions scenarios, ECS, income levels, parametric uncertainty, and model choice. We have also identified reasonable alternatives to the assumptions and modeling currently in use. As such, future analysis could expand the evaluation and consideration of alternatives consistent with the state of scientific knowledge to increase the robustness of results.

Given our interest in model behavior, we have said little about discounting up to this point. Discounting aggregates model responses over time. Economics can provide guidance given the type of investment and context, but selecting a discounting approach is complicated by many considerations (National Academies of Sciences Engineering and Medicine, 2017; Rose, 2012). One practical analytical issue is consistency with assumed economic growth, with lower (higher) economic growth implying a lower (higher) discount rate; and, uncertainty in economic growth implying uncertainty in the discount rate. The National Academy of Sciences identified this issue as well and recommended an approach to incorporate these elements (National Academies of Sciences Engineering and Medicine, 2017). Note that, while having alternative discounting schemes is practical, it creates a need for guidance on how the set of resulting SCCs should be utilized in regulatory analyses (Rose and Bistline, 2016). 
There are many alternatives to the choices associated with the USG experimental design. We have identified some, including input assumptions, climate models, discounting, and model and scenario weighting. Properly evaluating alternatives and comprehensively incorporating uncertainties is a research program onto itself and should be a policy priority. Our analyses have also identified a set of issues (e.g., implementation inconsistencies, arbitrary structural differences, dependence, and specific features needing justification), as well as model tendencies that need further evaluation. Together, these items call into question equal weighting of models and scenarios, and could justify different weighting schemes and/or different modeling altogether. Table 2 illustrates the implications of simply doing the former, weighting the current results differently. Other issues associated with the models and experimental design are still present.

Specifically, for Table 2, we assembled alternative distributions from the 150,000 estimates underlying the 3\% 2020 USG SCC values (Fig. 1) by first giving a zero weight to results based on the fifth socioeconomic/emissions projection, which is below current global emissions; and second, varying the weights given to results from individual models, from $100 \%$ to $0 \%$ weight for a single model with the remaining models weighted equally. The USG official estimates are also shown for comparison ("USG SCCs"). Table 2 includes 5th, as well as 95th, percentile values to provide symmetrical information about the tails of the distributions and better represent the uncertainty (National Academies of Sciences Engineering and Medicine, 2017). The table clearly illustrates the sensitivity of the current USG estimates to the weighting schemes. In particular, the individual models have pronounced roles, with PAGE pulling the multimodel average significantly higher, and DICE and FUND pulling it lower. This dichotomy demonstrates the importance of understanding, communicating, and justifying differences in models, as well as the individual model formulations and tendencies.

Finally, we note that consideration of alternatives to the multi-model approach would be practical. With multiple IAMs, it will always be challenging to ensure comparable and robust results that account for dependency. In the current SCC simulation context, where optimization or equilibrium is not required, one could design a framework component by component, choosing the best approach for each and

Table 2. SCC based on alternative weighting of $20203 \%$ discount rate USG values.

\begin{tabular}{lcccccccc}
\hline & USG SCCs & \multicolumn{6}{c}{ Without 5th socioeconomic/emissions results } \\
\cline { 2 - 8 } & & All & DICE & FUND & PAGE & DICE/FUND & DICE/PAGE & FUND/PAGE \\
\hline Average & $\$ 42$ & $\$ 44$ & $\$ 39$ & $\$ 21$ & $\$ 71$ & $\$ 30$ & $\$ 55$ & $\$ 46$ \\
5th percentile & - & $\$ 3$ & $\$ 16$ & $\$ 3$ & $\$ 5$ & $\$ 1$ & $\$ 7$ & $\$ 1$ \\
95th percentile & $\$ 123$ & $\$ 130$ & $\$ 76$ & $\$ 59$ & $\$ 297$ & $\$ 71$ & $\$ 183$ & $\$ 183$ \\
\hline
\end{tabular}


entertaining uncertainty through a single structure within each component. Such an approach offers full experimental control, statistically comparable results, and greater transparency regarding modeling and uncertainty. Furthermore, the framework could evolve over time with improved understanding of damages and feedbacks.

\section{Conclusion}

This study elucidates and assesses the modeling and raw detailed results underlying USG SCC estimation. We conduct the first detailed diagnostic and inter-comparison of the models used by the USG and others, isolating the socioeconomic, climate, and damage components, elucidating model structure, and presenting intermediate and comparable outputs not previously available. Together they provide insights into differences in SCC distributions between models. The distribution and average of SCC results from an individual model reflect a combination of responses across the components of the causal chain (recall Fig. 1). Through decomposition and comparison, our analysis provides insights into each model's results and relative differences.

From our detailed results and understanding of model structure, we are able to go even further and identify the key modeling elements behind each model's behavior and results, thereby enhancing understanding and enabling further scientific assessment. Overall, across models, we find significant differences in structure, implementation, and model behavior, and identify fundamental scientific issues with the SCC models and the current USG approach and estimates. The issues point to several opportunities for improving SCC estimation and increasing transparency and scientific and public confidence in results.

Going forward, it is important to communicate, evaluate, and justify differences and address those with insufficient scientific rationale, improve the representation of uncertainty in its various forms and the resulting robustness of estimates, and enhance documentation, including providing intermediate and disaggregated reference and incremental results over time from individual components and models. Taken together, our observations also suggest that the current multimodel approach be reconsidered. Finally, peer review of existing and future frameworks (e.g., models, runs, aggregation), uncertainties (standardized, model specific, and specifications), and other elements would be pragmatic and valuable. The current USG approach has been in place for some time and the latest estimates and/or models may be as well at some jurisdictional level (US federal, state, non-US federal), thus it is important that the public have confidence in the methods and numbers. ${ }^{10}$

Regarding uncertainty, it is well known that the SCC is sensitive to assumptions, which simply means that it is essential to explicitly incorporate uncertainty for those factors to which the SCC is sensitive. A challenge however is defining distributions for

\footnotetext{
${ }^{10}$ Note that National Academies of Sciences Engineering and Medicine (2017) was not asked to formally review or critique the current approach, though it did consider the approach in making recommendations.
} 
inputs and parameters. One must first identify potential values and then assign probabilities. In some cases, there is limited if any information to inform either, especially the latter. Even expert elicitation, which is sometimes used to develop distributions from expert judgement in lieu of gaps in observations and analysis, is simply a method for characterizing what is known and is therefore constrained by the state of knowledge (National Academies of Sciences Engineering and Medicine, 2017).

By providing a detailed technical foundation for better understanding estimation of the SCC, we hope this study encourages discussion and facilitates a new generation of SCC analyses and climate change research. In addition, it is important to look beyond the USG exercise and apply these findings more broadly to the representation of climate and damage components in IAMs for future research on climate risk management and global carbon policy. Damage estimation is an area particularly ripe for improvement given that current formulations are based on dated and dependent assumptions, and poorly understood calibrations. Finally, while this study focuses on SCC estimation, SCC application in calculating climate and net benefits is a separate, but important, topic, with guidance needed to avoid misapplication (Rose and Bistline, 2016).

\section{References}

Anthoff, D and RSJ Tol (2013). FUND v3.7 Scientific Documentation. Available at http:// www.fund-model.org/.

Bindoff, NL et al. (2013). Detection and attribution of climate change: From global to regional. In Climate Change 2013: The Physical Science Basis. Contribution of Working Group I to the Fifth Assessment Report of the Intergovernmental Panel on Climate Change. Cambridge: Cambridge University Press.

Clarke, L et al. (2009). International climate policy architectures: Overview of the EMF 22 International Scenarios. Energy Economics, 31, S64-S81.

Clarke, L et al. (2014). Assessing transformation pathways. In Climate Change 2014: Mitigation of Climate Change. Contribution of Working Group III to the Fifth Assessment Report of the Intergovernmental Panel on Climate Change, O Edenhofer et al. (eds.) Cambridge, UK, New York, NY, USA: Cambridge University Press. Available at https:// tntcat.iiasa.ac.at/AR5DB, pp. 413-510.

Field, C et al. (2014). Climate Change 2014: Impacts, Adaptation, and Vulnerability. Contribution of Working Group II to the Fifth Assessment Report of the Intergovernmental Panel on Climate Change. Cambridge: Cambridge University Press.

Hope, C (2011). The PAGE09 integrated assessment model: A technical description. Working Paper 4, Cambridge Judge Business School.

Howard, P (2014). Omitted damages: What's missing from the social cost of carbon. The Cost of Carbon Project of the Environmental Defense Fund, the Institute for Policy Integrity, and the Natural Resources Defense Council. March. Available at: http://costofcarbon.org/reports (last accessed May 28, 2017).

IPCC (2007). Climate Change 2007: Impacts, Adaptation and Vulnerability. In Contribution of Working Group II to the Fourth Assessment Report of the Intergovernmental Panel on 
Climate Change, ML Parry, OF Canziani, JP Palutikof, PJ van der Linden and CE Hanson (eds.). Cambridge, UK: Cambridge University Press.

Johnson, LT and C Hope (2012). The social cost of carbon in US regulatory impact analyses: An introduction and critique. Journal of Environmental Studies and Sciences, 2(3), 205-221 doi: 10.1007/s13412-012-0087-7.

Joos, E et al. (2013). Carbon dioxide and climate impulse response functions for the computation of greenhouse gas metrics: A multi-model analysis. Atmospheric Chemistry and Physics, 13, 2793-2825.

Meinshausen, M et al. (2009). Greenhouse-gas emission targets for limiting global warming to $2{ }^{\circ}$ C. Nature, 458, 1158-1163.

Meinshausen, M, S Raper and T Wigley (2011). Emulating coupled atmosphere-ocean and carbon cycle models with a simpler model, MAGICC6 - Part 1: Model description and calibration. Atmospheric Chemistry Physics, 11, 1417-1456.

National Academies of Sciences, Engineering, and Medicine (2017). Valuing Climate Damages: Updating Estimation of the Social Cost of Carbon Dioxide. Committee on assessing approaches to updating the Social Cost of Carbon, Board on Environmental Change and Society, Division of Behavioral and Social Sciences and Education. Washington, DC: The National Academies Press. doi: 10.17226/24651.

Nordhaus, W (2010). Economic aspects of global warming in a post-Copenhagen environment. Proceedings of the National Academy of Sciences, 107(26), 11721-11726.

Nordhaus, WD and J Boyer (2000). Warming the World: Economic Models of Global Warming. Cambridge: MIT Press.

Rose, SK (2012). The role of the social cost of carbon in policy. WIREs Climate Change, 3 , 195-212. doi:10.1002/wcc.163.

Rose, SK et al. (2014). Understanding the Social Cost of Carbon: A technical assessment. EPRI Report \#3002004657. Palo Alto, CA.

Rose, SK and J Bistline (2016). Applying the Social Cost of Carbon: Technical considerations. EPRI Report \#3002004659. Palo Alto, CA.

Tol, R (2009). The economic effects of climate change. Journal of Economic Perspectives, 23, 29-51.

United Nations (2015). Probabilistic population projections based on the world population prospects: The 2015 revision. Population Division, DESA. Available at http://esa.un.org/ unpd/ppp/.

Urban, NM et al. (2014). Historical and future learning about climate sensitivity. Geophysical Research Letters, 41(7), 2543-2552.

United States Government Accountability Office (2014). Regulatory Impact Analysis: Development of Social Cost of Carbon Estimates, Report to Congressional Requesters, GAO-14663, July 2014. Available at http://www.gao.gov/products/GAO-14-663 (last accessed May 29, 2017).

United States Government Interagency Working Group on Social Cost of Carbon (2010). Appendix 15A. Social Cost of Carbon for Regulatory Impact Analysis Under Executive Order 12866, March.

United States Government Interagency Working Group on Social Cost of Carbon (2013). Technical Update of the Social Cost of Carbon for Regulatory Impact Analysis Under Executive Order 12866, May.

United States Government Interagency Working Group on Social Cost of Carbon (2015). Technical Update of the Social Cost of Carbon for Regulatory Impact Analysis Under Executive Order 12866, July. 
United States Government Interagency Working Group on Social Cost of Greenhouse Gases (2016). Technical Update of the Social Cost of Carbon for Regulatory Impact Analysis Under Executive Order 12866, August.

United States Government Interagency Working Group on Social Cost of Greenhouse Gases (2016). Addendum to Technical Support Document on Social Cost of Carbon for Regulatory Impact Analysis under Executive Order 12866: Application of the Methodology to Estimate the Social Cost of Methane and the Social Cost of Nitrous Oxide, August.

van Vuuren, DP et al. (2011). The representative concentration pathways: An overview. Climatic Change, 109, 5-31. 